\title{
Risque, assurance et irréversibilité
}

Andràs November et Valérie November

\section{OpenEdition}

\section{Journals}

Édition électronique

URL : http://journals.openedition.org/ress/475

DOI : $10.4000 /$ ress.475

ISSN : 1663-4446

\section{Éditeur}

Librairie Droz

\section{Édition imprimée}

Date de publication : 1 novembre 2004

Pagination : 161-179

ISBN : 2-600-00980-9

ISSN : 0048-8046

Référence électronique

Andràs November et Valérie November, «Risque, assurance et irréversibilité », Revue européenne des sciences sociales [En ligne], XLII-130 | 2004, mis en ligne le 13 novembre 2009, consulté le 19 avril 2019. URL : http://journals.openedition.org/ress/475 ; DOI : 10.4000/ress.475 


\title{
RISQUE, ASSURANCE ET IRRÉVERSIBILITÉ
}

\begin{abstract}
Risques et assurances sont étroitement liés. D'abord, parce qu'on ne saurait concevoir les assurances sans les notions de risque et de sinistre. Ensuite, les risques très tôt ont été appréhendés sous forme d'assurance. Malgré cette liaison étroite, ces deux concepts ont été pris en charge par des communautés scientifiques distinctes, qui ont développé leur propre littérature et concepts. D'un côté, la science actuarielle qui se concentre sur les dimensions économiques et financières des risques. D'un autre, ce qu'on pourrait appeler «la science des risques » si elle existait, c'est-à-dire les sciences environnementales, de l'ingénierie et les sciences sociales qui s'intéressent aux dimensions avant tout environnementales, techniques et sociétales du risque. Face aux nouvelles formes que prennent actuellement les risques, particulièrement ceux liés aux biotechnologies, les régimes d'action auxquels appartiennent ces deux notions changent. Cet article tente d'esquisser les contours de ce changement et de pointer les obstacles à surmonter.

Nous déroulerons notre propos en cinq étapes ${ }^{1}$. Après avoir relevé les dimensions les plus caractéristiques de la notion de risque et de la relation risque-assurance usuelle, nous plongerons dans la confection du système assurantiel pré-moderne pour arriver aux mécanismes utilisés actuellement. Le recours au principe de précaution sera alors présenté en tant qu'illustration d'une possible nouvelle articulation de la relation risque-assurance.
\end{abstract}

\section{L'ASSURANCE : UNE TRADUCTION DU RISQUE}

Le risque - indissociable des décisions et activités humaines - constitue le socle des assurances, dont le but est d'abord de couvrir les dommages qui peuvent en résulter (assurances dommages) et d'indemniser, ensuite, le cas échéant, les préjudices causés à autrui suite à la faute imputable à son assuré (assurance responsabilité civile - $\mathrm{RC})^{2}$. En d'autres mots, la «société du risque», pour

Cet article se base sur des recherches menées depuis plusieurs années d'un côté par Andràs November sur les technologies nouvelles et le développement durable, de l'autre par Valérie November sur les risques, les controverses scientifiques et la surveillance à distance. Les auteurs remercient Elisabeth Rémy pour ses commentaires éclairants sur une version antérieure du manuscrit.

2 En effet, l'assurance est «l'opération par laquelle l'assureur s'engage à fournir une prestation au profit de l'assuré en cas de réalisation d'un événement aléatoire (le risque) en contrepartie d'une somme d'argent, la prime ou cotisation». L'Encyclopaedia Universalis (CD-Rom, version 8, 2002). 
reprendre le qualificatif désormais célèbre de Ulrich Beck ${ }^{3}$, est en même temps une «société assurantielle», terminologie utilisée par François Ewald pour caractériser la prolifération des assurances ${ }^{4}$.

C'est dans ce sens que s'établit une relation étroite entre risques et assurances. Le lien se noue autour de l'inquiétude que provoquent les risques et l'aspiration à la sécurité qu'apporte l'assurance. Il apparaît qu' «un des fondements du recours à une assurance est la crainte de la réalisation d'un certain aléa contre lequel on veut être protégé. [...] Plus un agent économique a peur d'un événement, plus son consentement à payer dans le contrat d'assurance est élevé ${ }^{5}$. Répondant à une exigence viscérale de conforter la sécurité dans la société, les assurances engendrent l'antidote du risque: dans la société techno-industrielle, l'aversion face aux risques va de pair avec la propension croissante à renforcer la couverture que procure l'assurance. Il serait toutefois injuste de ne mentionner que l'aversion de «l'agent économique» ou son désir de confort. Un grand nombre de contraintes juridiques obligent en effet chaque citoyen de s'assurer contre une série de risques qu'il le veuille ou non, augmentant par là de manière considérable la dépendance face aux assurances. C'est le cas du risque incendie, des assurances bâtiments, de la responsabilité civile et bien d'autres encore. En quelque sorte, le système assurantiel actuel (que la souscription soit obligatoire ou non) peut être vu comme l'expression en termes compensatoires des manifestations du risque (quels qu'ils soient). Il est donc possible de considérer que l'assurance est une forme de traduction du risque.

Cette relation, qui semble évidente au premier abord, n'exclut cependant pas le fait que le couple risque/assurance est traversé par de nombreuses tensions et oppositions. Nous détaillons ci-dessous cinq éléments particuliers de cette relation.

- Une relation probabiliste: d'une manière générale, pour faire face aux risques qui sont potentiels ou aléatoires, les assureurs cherchent à les objectiver, à les probabiliser et à les codifier (sur le plan juridique) en recourant à diverses techniques statistiques et aux calculs actuariels. L'application de ces outils permet d'établir une meilleure anticipation des risques assurés. L'assurance se fonde pour cela sur les lois probabilistes et les séries statistiques et peut être considéré comme «une machine à comptabiliser les risques».

- Une relation économique: on parle de risques lorsqu'il y a des incertitudes quant aux dommages qui pourraient survenir. Il est annonciateur d'un événement défavorable, souvent grave, dont on ne connaît ni le moment de surve-

\footnotetext{
Ulrich Beck, La société du risque. Sur la voie d'une autre modernité, Paris, Aubier, 2001 (première édition en allemand, 1986).

${ }^{4}$ François Ewald, Histoire de 1'Etat-Providence, Paris, Grasset, 1996. D'autres travaux font la liaison entre le phénomène assurantiel et la notion de risque. Voir notamment les recherches des économistes Henri Loubergé «Development in risk and insurance economics: The past 25 years,» in Dionne G. (Ed.), Handbook of Insurance Economics, Boston, Kluwer Academic Publishers, 2000, 3-33; et de Orio Giarini, Les limites du certain, Lausanne, Presse polytechnique et universitaire romande, 1990. Il faut également mentionner l'ouvrage de Godard Olivier, Henry Claude, Lagadec Patrick et al., Traité des nouveaux risques, coll. Folio-actuel, Paris, Gallimard, 2002, qui consacre une large part aux assurances (pp. 361-549).

s $\quad$ Godard et al., op. cit., p. 377.
} 
nance, ni l'ampleur. L'assurance, de son côté, cherche à établir des causalités qui sont les sources des sinistres, et à évaluer préalablement le montant des dommages possibles. En d'autres termes, l'assurance représente la dimension économique du risque, puisqu'elle s'attache surtout aux dommages qui en résultent $^{6}$. Comme l'assurance comprend toujours une contrepartie monétaire (prime d'assurance), elle s'apparente souvent, mais pas toujours, à une transaction économique à but lucratif.

- Une relation évolutive : les risques sont évolutifs, ils changent en fonction du développement technologique et de la complexification de l'organisation de la société, tandis que les assurances ne font que suivre cette évolution. Dans la civilisation techno-industrielle, le risque est non seulement omniprésent, mais aussi proportionnel à la puissance des technologies. Il nécessite sans arrêt l'élaboration de nouvelles connaissances scientifiques et techniques. Les nouvelles technologies - telles les biotechnologies - sont porteuses de risques nouveaux que le système d'assurances s'efforce d'appréhender et de prendre en charge, en inventant toujours de nouvelles formes d'assurances novatrices.

- Une relation limitée: ce sont les risques conventionnels (ou opération standard) connus, statistiquement prévisibles, qui constituent le principal domaine d'activité des assurances. Par contre, les nouveaux types de risques (appelés aussi «risques hypothétiques»), que les assurances n'arrivent que difficilement à appréhender ou anticiper, atteignent les limites de l' «assurabilité » à cause de la difficulté d'évaluer leurs conséquences éventuelles ${ }^{7}$. Dans ces cas, les assurances sont contraintes de remettre en cause la validation principale qui conduit à identifier les risques par l'intermédiaire de calculs de probabilité. Depuis quelques décennies, les risques à grande échelle font leur apparition et remettent en cause le système bien rodé des assurances traditionnelles, comme nous le verrons plus loin.

\section{LA MUTATION DES ASSURANCES}

Les premières assurances étaient les «sociétés mutuelles», qui survivent encore aujourd'hui ${ }^{8}$. En France par exemple, selon le Code de la mutualité, qui

$6 \quad$ Pour clarifier la terminologie utilisée ici, notons que le danger est une «menace qui peut compromettre la sûreté ou l'existence d'une personne ou d'une chose». Le risque est un «danger éventuel plus ou moins prévisible», tandis que le terme aléa désigne un «événement imprévisible» (Dictionnaire Le Petit Robert).

7 Cf. Pierre Lascoumes (1996, p. 363) qui distingue les risques reportés («ceux pour lesquels l'état des connaissances à un moment donné ne permet pas d'anticiper des effets dommageables qui se manifesteront à moyen ou long terme») et les risques résiduels («les risques connus mais qui en deçà d'un certain seuil ne peuvent plus être rationnellement appréhendés »).

«Assurance», in Encyclopaedia Universalis (CD-Rom, version 8, 2002). La première assurance connue dans l'histoire fut fondée par les tailleurs de pierre en Egypte, vers 1400 av. J.-C. Elle devait venir au secours de ses membres en cas d'accident. Peter Bernstein (1998, pp. 86 et 87) fait remonter l'origine des assurances au Code d'Hamourabi (1800 av. J.-C.) «qui consacre 282 arrêts aux hypothèques consenties sur un navire pour fournir les fonds nécessaires à son voyage. Aucune prime n'est versée, et si le bateau sombre, nul n'est tenu de rembourser les sommes avancées ». 
vient d'être modernisé, les sociétés mutuelles d'assurance se définissent comme les « groupements de personnes de droit privé à but non lucratif » qui, au moyen des cotisations de leurs membres, se proposent de mener une action de prévoyance, de solidarité ou d'entraide visant notamment la prévention des risques et la prise en charge commune de leurs conséquences. Leur but est de contribuer au «développement culturel, moral et physique et à l'amélioration des conditions de vie de leurs adhérents ${ }^{10}$. Ces mutuelles offrent à leurs membres un large éventail de services (notamment assurances maladie, incendie, accidents, responsabilité civile, assurances contre les calamités agricoles, protection juridique, assistance aux personnes ou cautionnement). Elles ne cherchent pas la réalisation de bénéfices; leurs excédents sont intégralement distribués, après constitution des réserves légales. Les assurances mutuelles mettent l'accent sur la solidarité et pratiquent la gestion socialisée des risques. En agissant ainsi, elles font partie du secteur de l'économie sociale, qui se situe à l'opposé de l'économie marchande ${ }^{11}$.

Un autre type d'assurance apparaît au Moyen Age, lié au développement du commerce maritime. Elle est basée sur un contrat dénommé «lettre de change maritime» ou "prêt à la grosse aventure». Il s'agit d'un emprunt gagé sur les marchandises transportées par voie maritime. Si les marchandises étaient perdues en route, le prêteur perdait tout droit au remboursement de la somme prêtée. Dans le cas contraire, celui-ci recevait son capital en retour, augmenté d'un intérêt appréciable.

\section{L'histoire de Zaccaria, négociant génois}

Pour illustrer ce cas de figure, on évoque souvent «l'histoire de Zaccaria», négociant génois qui flaira et réalisa une affaire juteuse, en 1298. Il apprit que les artisans de Bruges étaient demandeurs d'un produit utilisé dans l'industrie textile, qui était disponible à Venise. Toutefois, pour réaliser cette transaction, il devait emprunter la somme nécessaire pour acheter la marchandise et l'acheminer à Bruges par voie maritime. Cette affaire comportait de nombreux aléas, notamment le risque de fortune de mer, le risque de prix et le risque de perte de la cargaison. Rusé comme il l'était, il s'adressa à deux personnes fortunées qui acceptèrent de lui prêter la somme nécessaire et de fournir la couverture des risques, à un taux d'intérêt élevé. Dans cette opération, Zaccaria réussit à faire coup double: obtenir un emprunt et transférer simultanément ses risques à ses créanciers. Grâce à ce montage habile, Zaccaria put vendre une marchandise qui ne lui appartenait pas (pendant la transaction, elle était la propriété de ses bailleurs de fonds) et tirer des bénéfices de l'opération. Dans cette forme

$9 \quad$ Code de la mutualité, Journal officiel (République française), le 12 mars 2002. Il existe environ 5500 mutuelles en France (essentiellement dans le domaine de la santé), dont certaines ont des effectifs importants, comme les mutuelles des fonctionnaires de l'Education nationale, des PTT et des hospitaliers.

10 Il ne faut pas confondre les «sociétés d'assurance à forme mutuelle», qui sont des sociétés anonymes à caractère commercial, avec les «sociétés de mutuelle d'assurances », fondées sur les principes de la solidarité. Cf. «L'économie sociale entre étatisation et capitalisme», Cahiers français, n⿳o 221, mai-juin 1985 .

11 Cf. Bernard Eme et Jean-Louis Laville, «L'économie solidaire», Le Monde, 18 novembre 1992. L'économie solidaire a pour but de resituer l'économie dans son contexte social. Il s'agit d'une forme intermédiaire entre le capitalisme et l'étatisme. 
d'assurance précapitaliste, les germes de l'étape capitaliste étaient déjà présents du fait qu'assurance et investissement financier étaient étroitement liés.

Source: Giraud Pierre-Noël, «L'histoire de Zaccaria, négociant génois », Le commerce de promesses, Paris, Seuil, 2001, pp. 35-73.

Le système moderne des assurances se met en place dès la révolution industrielle et le développement rapide des assurances modernes est directement lié à l'essor du capitalisme. C'est à partir de cette époque que «les sociétés sont devenues des manufactures de risques» ${ }^{12}$, dont le nombre et la gravité s'intensifiaient. Parallèlement, les assurances assument un rôle primordial dans la préservation $d u$ patrimoine $^{13}$ individuel et collectif, privé et public, que l'avènement du risque menace, tout en permettant de maintenir les activités économiques malgré l'adversité du sinistre. On peut observer que, appliquant des méthodes traditionnelles, les assureurs mettent en œuvre des instruments standardisés, économétriques, fragmentés et probabilistes que la survenue et l'ampleur des risques catastrophiques remettent fondamentalement en cause. Avec l'évolution de la nature des risques à couvrir, les assureurs ont été amenés à élaborer des méthodes toujours plus sophistiquées et complexes pour appréhender les conséquences financières des dommages. Dans ce dessein, ils ont largement puisé dans les théories de la modélisation ou des scénarios.

Actuellement, deux indicateurs de base constituent les principaux instruments de l'aide à la décision pour élaborer les contrats d'assurance correspondant aux différents types de dommages: le sinistre raisonnablement escomptable (SRE), qui peut se réaliser dans les conditions normales d'activité en l'absence d'événements exceptionnels ou accidentels, le sinistre maximum possible (SMP) intervient dans les circonstances les plus défavorables (worst case) qui sont exceptionnellement réunies. Les SRE et SMP permettent de mesurer le potentiel de réalisation des risques. Dans le cas des tremblements de terre par exemple, la quantification de «l'exposition au tremblement de terre» commence par l'établissement d'un inventaire de tremblements de terre d'une région donnée, comprenant la fréquence de l'occurrence des sinistres, leur intensité (fondée sur les observations historiques qui sont traduites sur une échelle à douze degrés) et magnitude (enregistrée par le sismographe et reportée à l'échelle de Richter). Partant de ces données, il devient possible de quantifier l'exposition au danger en déterminant les périodes de récurrence des tremblements de terre et de calculer la probabilité des dommages. Les conditions d'assurance seront définies en fonction de ces calculs. Elles porteront sur les risques assurés, les limites de la garantie, les limites par événement.

12 Bruno Latour, «Beck ou comment refaire son outillage intellectuel», Préface, in Beck Ulrich, La Société du risque, op. cit., p. 8.

13 Jean Baechler, Le capitalisme, t. II, L'économie capitaliste, coll. Folio-histoire, Paris, Gallimard, 1995. Par ailleurs, nous entendons par patrimoine «l'ensemble des biens corporels et incorporels d'une personne (physique ou morale) ou d'un groupe de personnes » (Le Petit Robert). Cette définition ne couvre pas, et de loin, la complexité de la notion. Pour François Ost, La nature hors la loi. L'écologie à l'épreuve du droit, Paris, La Découverte, 1995, «le patrimoine est le statut juridique pour le milieu» (pp. 306-337). Nous avons opté pour la notion de patrimoine qui englobe les biens matériels, publics (environnement) et symboliques. 


\section{L'ÉCONOMIE DES RISQUES}

L'économie des risques englobe l'ensemble des transactions entre assureurs et assurés dont le but est la couverture des dommages qui découlent des risques, contre le paiement d'une prime ${ }^{14}$. La source de la rentabilité des assurances est la différence entre le montant des primes perçues, d'une part, et indemnités déboursées pour les dommages, d'autre part. Basées sur la réalisation aléatoire des risques, les assurances peuvent atteindre une rentabilité élevée tant que les indemnités des dommages sont relativement limitées. En cumulant les excédents des primes et des réserves (légales) au cours des années fastes pendant lesquelles elles enregistrent peu de sinistres, les sociétés d'assurances sont devenues des institutions financières puissantes. Au fil des décennies, l'industrie de l'assurance a acquis un véritable pouvoir financier.

En réalité, l'assurance encadre les risques avant (ex ante) et après (ex post) la survenance d'un sinistre ${ }^{15}$. Mais son rôle primordial est d'intervenir quand l'événement assuré se produit et de dédommager ses conséquences. Autrement dit, le risque devient réel au moment où le dommage paraît. C'est la concrétisation des risques qui constitue le véritable enjeu des assurances. En prenant en charge les coûts des dommages, l'assurance établit le lien monétaire entre le risque potentiel (virtuel et théorique) et le risque manifesté (le sinistre). C'est précisément le point d'ancrage économique des risques.

Les assureurs participent aussi à la prévention des risques. Ils apprivoisent le risque, préparent les acteurs à son avènement qu'elles rendent prévisible, présentable ou acceptable. Certes, l'assurance encourage à vivre avec le risque, puisqu'elle promet de réparer les dommages, mais en même temps, elle dissuade de prendre des risques inconsidérés et incite les assurés à observer un comportement prudent afin d'éviter les sinistres ${ }^{16}$.

Face aux risques les plus connus et les plus fréquents, l'assurance répartit «l'incertitude» entre les différents porteurs de risques, en garantissant l'indemnisation des dommages ${ }^{17}$. Pour être en mesure de couvrir les risques, les assureurs recherchent la distribution optimale des coûts entre les assurés concernés par le même risque (par exemple, les automobilistes), ou par le transfert des charges des sinistres à des investisseurs non directement impliqués, mais intéressés par un gain financier qu'offrent les compagnies d'assurances. Les cotisations (primes) prévues en contrepartie de la prestation des assureurs sont également distribuées entre tous les preneurs d'assurance en appliquant la loi des grands nombres.

14 Selon les estimations, le niveau des primes des assurances directes atteint 2'500 milliards d'euros.

15 Dans le domaine des assurances, la notion de dommage se définit comme «une atteinte portée à l'intégrité d'une chose ou d'une personne; en même temps, le dommage peut être corporel, matériel ou immatériel». Quant au dommage écologique, il est «l'atteinte irréversible ou un (grave) dommage causé au milieu naturel, dont la restauration ne peut être réalisée qu'à long terme, ou encore une perturbation de l'équilibre naturel». Swiss Re, L'environnement, la responsabilité civile et l'assurance, Zurich, 1996, p. 6.

16 On parle aussi de «risque moral», lié à la négligence d'un assuré de se protéger contre le risque assuré.

17 Swiss Re, «Transfert alternatif des risques (ART): état des lieux », Sigma, no 1, 2003, p. 15. 
Dans certains cas, les assureurs - en particulier les compagnies de réassurances - exercent une pression sur leurs assurés afin qu'ils adoptent un comportement responsable permettant de réduire les risques dont les conséquences peuvent être socialement et écologiquement lourdes. Par exemple, la Compagnie de Réassurances (Swiss Re) de Zurich, s'est adressée récemment aux grandes entreprises afin de les inciter à adopter des méthodes de production permettant de réduire leurs émissions de gaz à effet de serre, en menaçant les récalcitrants d'une augmentation considérable de leurs primes. Dans ces cas, l'assureur prend des mesures actives d'information ou de prévention; il peut en dernier recours refuser de couvrir certains risques. L'attitude interventionniste des assureurs est dictée par la nécessité. En effet, les grandes assurances constatent que le coût des catastrophes a subi une forte hausse depuis plusieurs décennies. Cette évolution entraîne l'augmentation des sommes déboursées pour les indemnités qui entraîne les conséquences financières qui peuvent menacer leur solvabilité et provoquer même leur faillite. Par exemple, les coûts des 350 catastrophes naturelles et techniques recensés en 2002 ont été particulièrement élevés. Selon les estimations des grandes compagnies d'assurances, les dommages se sont chiffrés à 65 milliards de francs (contre 40 milliards en 2001), dont 17 milliards ont été pris en charge par les assureurs ${ }^{18}$.

\section{LES RISQUES À GRANDE ÉCHELLE CHANGENT LA DONNE}

On parle de risques à grande échelle pour qualifier certains risques naturels et technologiques. Patrick Lagadec et Ulrich Beck, parmi d'autres, ont très bien décrit leurs caractéristiques. Ces «événements extrêmes » ${ }^{19}$ comme il est dit des catastrophes naturelles menacent de manière soudaine et grave aussi bien la population que l'environnement. En cela, ils induisent un changement d'échelle dans l'espace et dans le temps.

Le premier auteur, Patrick Lagadec, a inventé le terme de «risque technologique majeur» pour qualifier ces risques dont les conséquences sont potentiellement très importantes mais dont la probabilité de se produire est petite ${ }^{20}$. Ces nouveaux risques sont rares, mais susceptibles de se produire à n'importe quel moment et de provoquer de gros dégâts. Il s'agit de risques «proliférants » qui, s'ils se réalisent, agissent en multiplicateur, et leurs conséquences peuvent dépasser la capacité financière des assurances à indemniser les dommages. L'«effet domino » est particulièrement redouté.

Le second auteur, Ulrich Beck, montre que notre société évolue vers une société du «risque incompressible» dans laquelle ce mode de protection s'amoindrit paradoxalement à mesure que croît l'ampleur du danger. Face à ce type de risque, la collectivité pourrait devenir une «société sans assurances». Les dégâts

18 Tribune de Genève, le 17 décembre 2003.

19 Cf. OcCC (Organe consultatif sur les changements climatiques), Evénements extrêmes et changements climatiques, Berne, 2003.

20 Patrick Lagadec, La civilisation du risque: catastrophes technologiques et responsabilité sociale, Paris, Seuil, 1981. 
éventuels en jeu sont «illimités, globaux et souvent irréparables, ce qui enlève toute signification à l'idée de compensation monétaire ${ }^{21}$. De plus, ce même auteur relève que l'accident n'a plus de limites (spatiales et temporelles) et change de ce fait de signification: il devient un incident dont on voit le début mais non la fin, «une débauche de destructions rampantes, galopantes et se superposant les unes aux autres ». Or cela signifie qu'il n'y a plus de critères de normalité ni de méthodes de mesure et donc de base pour évaluer les dangers. On compare ce qui n'est pas comparable, l'évaluation devient dissimulation. Selon Beck, il y aurait confusion de deux siècles: les dangers auxquels nous sommes exposés sont de ce siècle et les moyens grâce auxquels on promet de les maîtriser pour assurer notre sécurité appartiennent à un autre siècle, héritage de la société industrielle du XIX et du début du $\mathrm{XX}^{\mathrm{e}}$ siècle.

La multiplication des gros risques catastrophiques naturels ou technologiques, pris en compte depuis une quarantaine d'années, pose de nouveaux défis aux assureurs et aux assurés ${ }^{22}$. Devant l'ampleur que peuvent prendre les catastrophes écologiques et technologiques, la limite de l'assurabilité de certains risques peut être atteinte. Ce sont les montants toujours plus élevés des indemnisations qui mettent en péril la capacité financière des assureurs ${ }^{23}$. Il n'est pas étonnant qu'une escalade des primes s'engage généralement à chaque survenance d'une catastrophe naturelle ou technologique. Suite à une catastrophe importante et en fonction des dommages subis, les assureurs décident de majorer leurs primes de $80 \%$ à 200\% (comme cela a été le cas par exemple après l'explosion de l'usine AZF à Toulouse). Les augmentations des primes pèsent lourdement sur la rentabilité des entreprises et les mettent devant un dilemme: ou bien elles acceptent les charges de plus en plus élevées qu'elles doivent débourser pour les risques, ou bien elles trouvent une nouvelle solution en matière de couverture de leurs risques.

L'exemple de la contamination des semences (non OGM) par StarLink, produit génétiquement modifié non autorisée pour l'alimentation humaine, illustre les répercussions multiples dans la chaîne alimentaire d'une erreur de manipulation $^{24}$. Un cas similaire, un OGM contenant un vaccin de porc qui s'est mélangé au soja, a coûté 3.75 millions de US dollars à la société ProdiGen de Texas, son fabriquant ${ }^{25}$.

21 Ulrich Beck, «De la société industrielle à la société à risques », Revue suisse de sociologie, no 19, 1993, pp. 316-319.

22 La liste des catastrophes (surtout naturelles et techniques) de la dernière décennie s'allonge chaque année: parmi les événements les plus coûteux et les plus meurtriers se classent l'ouragan Andrew aux Etats-Unis, en 1992 (19 milliards de dollars), et le tremblement de terre de Kobé au Japon, en 1995 (2,8 milliards de dollars). Par ailleurs, depuis 1960, le nombre des catastrophes naturelles a été multiplié par trois, le montant des dégâts économiques par neuf et celui des préjudices par quinze (Munich Re).

23 Dans la classification des assureurs, la catastrophe est un sinistre dont le montant des pertes assurées dépasse 25 millions de dollars, tandis que les dommages d'un cataclysme dépassent 5 milliards de dollars.

24 Cf. Gilles-Eric Séralini, Génétiquement incorrect, Paris, Flammarion, 2003, p. 264.

${ }_{25}$ Les deux exemples ont été exposés lors de International Biotech Forum, Conference on Risk Perception, Liability and Insurability, 3-4 November 2003, Rüschlikon, Swiss Re, Centre for Global Dialogue. 


\section{Le cas de contamination par StarLink ${ }^{\mathrm{TM}}$}

Dans le domaine de la biotechnologie végétale, les montants des dommages peuvent prendre rapidement une ampleur financière considérable, comme le montre le cas «StarLink». La marque «StarLink» désigne une variante de maïs Bt (Bacillus Thuringensis), résistante aux herbicides, développée par Aventis CropSciences (rachetée par la suite par Bayer), dont l'autorisation de dissémination était limitée à l'alimentation animale, et interdit par conséquent à l'alimentation humaine. Cette interdiction était motivée par la présence d'une protéine (Cry9C) soupçonnée allergène. En septembre 2000, une organisation non gouvernementale américaine (Genetically Engineered Food Alert - GEFA) a dénoncé la présence de «StarLink» dans les tortillas de Kraft Food. Il s'est avéré que la farine (non OGM) utilisée pour la fabrication du produit a été contaminée par «StarLink». Dans un premier temps, Kraft Food a retiré du marché 2,5 millions de paquets de tortillas. Par la suite, tous les fabricants de tortillas et de produits similaires (environ 300 produits..) ont dû également retirer leurs produits du marché. En outre, les semences contaminées ont du être brûlées. Selon les estimations, le coût de cet «accident» a dépassé un milliard de dollars US. Somme qui est considérée comme la plus élevée jusqu'à ce jour dans l'histoire des OGM.

Les entreprises industrielles multinationales s'exposent, par leur taille et leurs activités, à des risques élevés que les assureurs hésitent à couvrir ou, a contrario, les grandes entreprises renoncent à payer des primes toujours plus élevées pour s'assurer, estimant que ce capital pourrait être mieux investi. En réalité, les entreprises sont obligées d'assumer et d'assurer les multiples risques, car elles ne peuvent rester sans assurances: un accident important, dont elles seraient responsables et qu'elles devraient indemniser, pourra entraîner leur faillite. Dans le cadre de leur politique d'assurances des risques, elles doivent décider quels sont les risques qu'elles doivent assurer, à quel prix, avec quelle méthode et établir ainsi leur portefeuille d'assurances.

Pour pouvoir faire face aux nouveaux risques majeurs, l'industrie des assurances a été amenée à développer de nouvelles méthodes de prévention ou de transfert de risques. On peut observer que l'imagination des assureurs et les grandes entreprises exposées à des catastrophes technologiques et environnementales (comme l'industrie pétrochimique ou chimique) est sans borne dans l'invention de nouvelles formules d'évitement ou de couverture des risques à grande échelle. La prise en charge des nouveaux risques peut suivre plusieurs voies, notamment l'auto-assurance (ou self-financing) des entreprises, les risques syndiqués par plusieurs entreprises d'assurance et le transfert des risques aux marchés financiers.

- Plusieurs grandes entreprises ont décidé de se réunir et de s'auto-assurer, au lieu de recourir aux services des sociétés d'assurances. L'auto-assurance peut prendre plusieurs formes, tels les pools ou les captives qui permettent de partager ou de mutualiser les grands risques entre leurs adhérents. Ainsi, les assureurs transfèrent une partie des risques aux grandes entreprises multinationales. Par exemple, un grand groupe peut créer sa propre compagnie d'assurance et s'assurer auprès d'elle (assurance captive). Mais les compagnies d'assurances traditionnelles ne sont pas complètement écartées, elles resteront présentes dans le processus pour «accompagner» leurs clients comme consultants en risques. Une forme particulière d'assurance voit l'entreprise et l'assurance liées par un contrat de longue durée où les primes sont mises dans une caisse commune. En cas de sinistre, l'assuré est remboursé pour les dégâts subis, 
mais l'assurance garde les primes; dans le cas contraire, en absence de sinistre pendant une période déterminée, l'assuré peut récupérer une partie des primes.

Pour faire face aux risques majeurs, les compagnies de réassurances occupent une place prépondérante dans l'assurance de grands risques. Comme «assureurs des assureurs », elles constituent le dernier maillon de la couverture des risques et du financement des dommages survenus. Quelquefois la capacité financière d'une seule société est insuffisante et plusieurs compagnies doivent se regrouper en syndicat pour assurer les risques à grande échelle.

- Dans une démarche préventive, certaines compagnies d'assurances proposent à leurs clients - essentiellement aux grandes entreprises industrielles - des services fournis par un groupe de spécialistes (chimistes, ingénieurs, techniciens) capables de mettre le doigt sur les faiblesses d'organisation ou des procédés de production qui présentent des risques potentiels importants et d'établir un cahier de charge en matière de sécurité en amont ou d'organiser les secours rapides en cas d'accident.

- Dans les cas exceptionnels et à défaut de couverture financière des dommages importants, comme les inondations ou les tremblement de terre, l'Etat devient le «superassureur» ou payeur de dernier recours. Mais l'Etat a aussi un rôle actif à jouer. Il peut instituer l'assurance obligatoire (comme l'assurance incendie) ou octroyer des subventions en vue de favoriser l'assurance de certains risques ou promouvoir la prévention. En tout état de cause, l'intervention de l'Etat dans les domaines du risque et de la couverture des dommages résiduels ou non assurés se fait au nom de la solidarité nationale ${ }^{26}$.

- Enfin, quand les coûts prévisibles des catastrophes semblent mettre en danger l'équilibre financier des assureurs, ces derniers peuvent renoncer à assurer une entreprise qui présente des risques importants. C'est le cas des risques technologiques majeurs ou catastrophes imprévisibles, qui frappent avec soudaineté et dont les effets peuvent être irréversibles. Ce genre de risque est exclu généralement des contrats d'assurance. Par exemple, les grands assureurs comme Munich Re ou Swiss Re n'ont pas accepté d'assurer Monsanto et Novartis pour les risques de pollution génétique, simplement parce qu'elles ne savent ni évaluer ni chiffrer leurs conséquences financières ${ }^{27}$.

Une nouvelle phase s'ouvre dans les activités des assurances avec le transfert «alternatif» des risques (ART), qui combine les techniques d'assurance et les outils financiers ${ }^{28}$. Les assureurs et les réassureurs internationaux ont été particulièrement inventifs pour développer toute une série de services qui associent les instruments bancaires aux assurances, qu'ils offrent ensuite à leurs clients, généralement de grands groupes multinationaux, leur but étant de transformer l'assu-

26 Godard et al., op. cit., p. 447.

27 Aronson Daniel, «Les assureurs tirent la sonnette d'alarme», Alternatives économiques, nº 174, oct. 1999 , pp. 46-48.

28 Selon les estimations, le marché du transfert alternatif des risques a atteint 88 milliards de dollars en 2001 . 
rance d'un risque en divers produits financiers sophistiqués qu'ils proposent aux investisseurs ${ }^{29}$. Les nouvelles techniques de la réassurance financière, ont pour but de transférer les risques importants vers les marchés financiers. Elles englobent notamment les services financiers aux compagnies d'assurance, le financement de risques offert aux entreprises multinationales, le traitement des sinistres et la gestion des sociétés qui se chargent de l'auto-assurance des risques des entreprises multinationales (captives) ${ }^{30}$. Ces méthodes permettent de redistribuer le risque d'assurance entre un grand nombre d'actionnaires, qui achètent les papiers valeurs correspondants dans l'espoir de réaliser un profit. De transfert en transfert, la bourse devient ainsi le lieu central de la prise en charge des gros risques. Peu à peu, les assurances s'éloignent de leur métier initial d'assureur, pour endosser le rôle de spécialiste en ingénierie financière. Ce n'est plus le risque matériel qui est le souci principal des grands assureurs, mais le risque de capital. L'assureur s'efface derrière le financier et s'adonne aux montages financiers spéculatifs qui semblent relever de la «finance-fiction». En voici un exemple:

Swiss Re et Tokyo Marine ont signé le contrat de $s a^{31}$ aux termes duquel 550 millions de dollars de risques de tremblement de terre et de typhon au Japon ont été échangés contre des risques de tremblement de terre en Californie, d'ouragan en Floride et de tempête en Europe. Rendue possible par de récentes avancées en technologie de modélisation du risque, cette transaction signifie un profil de risque plus équilibré pour les deux partenaires et leur permet ainsi d'optimiser l'utilisation de leurs capitaux ${ }^{32}$.

Cet exemple montre que la réalité peut dépasser la fiction lorsque les assurances empruntent les instruments financiers spéculatifs développés par les banques. Il indique, que les assureurs ont mis en place un instrument qui permet d'échanger les contrats d'assurances garantissant la couverture de risques de tremblement de terre contre les contrats de même nature d'un autre assureur. La condition de base d'une telle transaction, connue sur les marchés financiers, est l'existence d'une institution (une bourse) où l'on peut effectuer l'échange des contrats qui sont traités comme d'autres papiers valeurs (actions ou obligations). La première bourse spécialisée dans la négociation des risques catastrophiques et dans le transfert des risques, le CATEX (Catastrophe Risk Exchange) a été créée en 1996 à New York. Il est ouvert aux porteurs des risques (assurances, réassu-

29 Le vocabulaire des assureurs fourmille de néologismes techniques, pratiquement incompréhensibles pour les non-initiés. En voici quelques exemples: finite risk, titrisation des risques, risques alternatifs, obligations de catastrophes (cat. bonds), portefeuille de défaut, dérivés financiers, etc. Les caractéristiques de ces différents services financiers sont présentées plus en détail par Godard et al., op. cit., pp. 412-431 et dans de nombreuses publications des grandes compagnies d'assurances (par ex. Munich Re, Swiss RE, Zurich, etc.).

Le raisonnement concernant la capacité d'absorption des marchés financiers est fondé sur l'observation que la volatilité journalière des bourses est identique à celle d'un tremblement de terre, soit 100 milliards de dollars... En d'autres termes, nous assistons à la création ou à la destruction virtuelle (selon l'évolution des cotations boursières) de valeurs financières. Cf. Swiss Re, Le génie génétique et l'assurance RC. Le poids de l'opinion publique, Zurich, 1998.

30 Swiss Re, Rapport annuel et Rapport de gestion, 2002.

31 Les swaps sont bien connus dans le domaine financier permettant aux banques de se prémunir contre les fluctuations des taux d'intérêt.

32 Swiss Re, Rapport annuel 2002 et Rapport de gestion, p. 10. 
rances, courtiers, sociétés captives des entreprises multinationales), dans le dessein de diversifier leur exposition aux risques ${ }^{33}$.

Mais le recours aux dérivés financiers ne débouche pas forcément sur un gain pour l'entreprise, car elle peut prendre une position sur le marché qui peut aboutir à une perte. Dans ce cas de figure, le risque original (matériel) est doublé par un risque financier pour l'assuré. C'est dans ce sens que «la nouveauté de ces instruments (financiers) rend l'incertitude (au sens de Keynes et Knight) qui leur est attachée plus inquiétante ${ }^{34}$. En fait, chercher la couverture financière aux risques catastrophiques par le biais des marchés financiers contribue à concevoir le risque comme s'il était une «chose», ou une marchandise. Avec la création de plus en plus étendue d'instruments financiers - les titres d'assurance (insurance linked securities) ou les obligations catastrophe (catastrophe bonds) - les (ré)assureurs deviennent l'un des principaux acteurs du capitalisme financier globalisé. Ils se transformant en institutions financières à but spéculatif ${ }^{35}$.

Il n'en reste pas moins que les différents montages financiers astucieux (et d'une grande complexité technique) constituent vraisemblablement l'ultime étape de l'assurabilité et de la couverture des gros risques.

\section{ASSURABILITÉ VERSUS IRRÉVERSIBILITÉ}

Le développement fulgurant des nouvelles technologies (en particulier le génie génétique) qui font apparaître «une tendance à l'extension infinie des risques » ouvre un nouveau chapitre dans la politique des assurances vis-à-vis des risques $»^{36}$. En effet, les nouveaux risques technologiques majeurs deviennent toujours plus incertains plus vastes et plus menaçants (situation dénommée «incertitude radicale»). Par exemple, les risques hypothétiques - parce qu'indéfinissables, insaisissables et imprévisibles - liés aux organismes génétiquement modifiés (OGM) posent des problèmes cruciaux aussi bien aux utilisateurs qu'aux assureurs $^{37}$.

Toutefois, il serait erroné d'envisager les risques uniquement sous l'angle technico-financier, car il faut aussi prendre en considération la perte immatérielle, qui est incommensurable ${ }^{38}$. Comme le note Lagadec, «le changement d'échelle des drames industriels rend la répartition financière difficile, voire impossible, ou

33 Voir Godard et al, op. cit. pp. 411-414, pour explications plus détaillées sur le CATEX.

${ }^{34}$ Godard et al., op. cit., p. 547. Voir également Christian Schmidt, «Risque et incertitude: une nouvelle interprétation», Risques. Les Cahiers de l'assurance, no 25, 1996, pp. 163-174.

35 La notion de «capitalisme financier» occupe, avec le capital marchand et le capital industriel, une place de choix dans la théorie économique du marxisme (et du léninisme). Elle constitue le stade avancé du capitalisme et désigne la l' interpénétration des institutions financières (banques) et de l'industrie.

36 Laurence Engel, La responsabilité en crise, Paris, Hachette, 1995, p. 7.

37 Les techniques d'ingénierie génétique permettent de modifier le patrimoine génétique des organismes vivants.

38 Nous renvoyons à cet égard à l'argument développé par Mark Hunyadi: «Comme par définition, le champ des risques possibles est incommensurablement plus grand que celui des risques avérés, 
simplement dépourvue de signification ${ }^{39}$. Mais au-delà des conséquences financières potentielles qui peuvent être élevées, voire incalculables, on doit prendre encore en compte des pertes matérielles et immatérielles irréversibles. Par exemple, on ne peut pas compenser par une valeur monétaire la pollution génétique par le transfert de pollen, la pollution environnementale des sols ou l'érosion lente et irrémédiable de la biodiversité. En fait, remarque Séralini, «personne ne peut plus supprimer l'ensemble des gènes artificiels que nous avons lâchés parmi les plantes cultivées $\gg^{40}$.

Il est intéressant de noter que la notion d'irréversibilité apparaît rarement dans les législations nationales. La loi Barnier (loi 95-1001 sur le renforcement de la protection de l'environnement de 1995) semble constituer une exception quand elle stipule que «l'absence de certitude ne doit pas retarder les mesures effectives et proportionnées pour prévenir des dommages graves et irréversibles ».

D’une manière générale, le terme irréversibilité désigne l'incapacité des acteurs à changer une situation ou le cours d'un processus »; tandis que le principe de précaution incite à décider et/ou à agir (soit d'une manière active, soit en s'abstenant) dans des conditions d'incertitude en se basant sur les hypothèses préalables à l'acquisition des connaissances scientifiques plus étendues ${ }^{41}$. Certes, l'irréversibilité est souvent présente dans les activités humaines, en particulier dans les choix scientifiques et techniques dont l'issue est recouverte par un «voile d'ignorance». Comme on peut constater, «irréversibilité » et «précaution»sont proches mais pas analogues et ne se situent pas sur la même échelle ${ }^{42}$.

En tout état de cause, avec la prise de conscience de l'irréversibilité dans l'application des biotechnologies, une étape importante est franchie dans le contrat socio-économique qui lie les assurances à la société et dans cette perspective les risques prennent une nouvelle dimension. La prise en compte des aspects socioculturels devient tout aussi cruciale que les aspects techniques: le tout joue un rôle déterminant dans la perception du public à l'égard des risques technologiques. Comme le note la publication d'un réassureur, «ce qui importe lorsqu'on est confronté au risque lié au génie génétique, ce n'est pas qu'il soit dangereux, mais dans quelle mesure il est considéré comme dangereux» ${ }^{43}$. Autrement dit, la perception et la tolérance de la société à l'égard des risques induits par les nouvelles technologies (en particulier des biotechnologies) subissent une muta-

on devrait prendre incommensurablement plus de mesures d'évitement pour les risques possibles que pour les risques avérés. On ne saurait caractériser plus précisément la paralysie totale de l'action » (in Esprit, n 8-9, août-septembre 2003, pp. 152-153).

39 Patrick Lagadec, in Demarcq François, «Le risque technologique majeur», Problèmes économiques et sociaux, $\mathrm{n}^{\mathrm{0}}$ 591, septembre 1988, p. 47.

40 «OGM et irréversibilité», in Gilles-Eric Séralini, Génétiquement incorrect, Paris, Flammarion. 2003, pp. 263-264.

${ }^{41}$ Cf. «La dialectique réversibilité-irréversibilité: une mise en perspective», introduction à l'ouvrage de Boyer Robert, Chavance Bernard, Godard Olivier, Les figures de l'irréversibilité en économie, Paris, EHESS, 1991. pp. 11-31. Voir aussi la contribution de Mark Hunyadi dans ce volume qui parle de «précaution abductive» et de «précaution pro-ductive»; cette dernière se définit par un raisonnement orienté vers le futur...

42 L'étude plus poussée des relations entre les notions d'irréversibilité et de précaution dépasse le cadre de cet article. Cette question mériterait d'être approfondie par un projet de recherche futur.

${ }_{43}$ Swiss Re, Le génie génétique..., op. cit., p. 8. (Souligné par les auteurs) 
tion. A cause de cette part d'irréversibilité, le risque dépasse les parties liées par le contrat entre assureurs et assurés, il concerne désormais tous les acteurs et toutes les institutions en occupant une place importante dans l'espace public.

Face à ces problèmes émergents, les assureurs se voient dépossédés de leur fonction primordiale qui est d'assurer les risques biotechnologiques. Ils observent l'apparition des risques auxquels ils ne peuvent plus offrir une couverture adéquate. Comme le note le Livre blanc de la Fédération française des sociétés d'assurances: «Comment assurer les risques liés aux OGM? C'est l'assurabilité des risques qui se trouve remise en question ${ }^{44}$. Or, ne pouvant plus garantir la prise en charge de toutes les conséquences des risques, les assurances se trouvent dans une impasse devant la menace potentielle des plantes transgéniques. La traduction d'un danger indéterminé en «risque» suit en effet un processus par lequel les compagnies d'assurances parviennent à déterminer l'occurrence d'un risque en appliquant les méthodes probabilistes et statistiques; elles mettent en face de ce risque une valeur monétaire du dommage qui pourrait survenir. Il est évident que cette démarche ne peut être suivie dans l'assurance des risques que peuvent présenter les OGM. «Assurer ou ne pas assurer», telle est la question que pose désormais avec une certaine candeur la société Swiss Re. Mis devant cette situation inconfortable, les assureurs sont amenés à reconsidérer l'assurabilité des risques biotechnologiques et la réparation des dommages $(\mathrm{RC})^{45}$.

Cette situation n'échappe pas aux institutions politiques et administratives qui mettent une pression sur les assureurs (privés et publics) afin qu'ils assument leur rôle et qu'ils offrent une couverture des risques biotechnologiques. C'est ainsi qu'un rapport du Commissariat Général du Plan français recommande aux autorités d'établir une politique d'assurance qui prend en compte des éventuels «risques de masse» liés aux $\mathrm{OGM}^{46}$. Un autre rapport, élaboré sous l'égide du Ministère français de l'écologie et du développement durable, constatant qu'aucun des essais des OGM au champ libre n'est couvert par l'assurance, recommande d'inscrire dans la loi «l'obligation d'assurance pour passer du stade des essais confinés à celui des essais en plein champ». En outre, les auteurs du rapport prévoient «la constitution d'un fond de solidarité par les opérateurs du secteur des biotechnologies » pour appuyer le secteur d'assurance ${ }^{47}$.

Actuellement, les assureurs insistent sur le fait que pour garantir un minimum d'assurance aux risques des biotechnologies végétales, diverses conditions préalables doivent être remplies: d'abord, la quantification du risque et l'évaluation des coûts prévisibles sont indispensables pour envisager l'assurance des risques

$44 \quad$ Le Monde, 6 octobre 2002.

45 Thomas Epprecht (Swiss Re), «Cartagena Protocol on Biosafety, Insurance Industry and Art 27 (Liability and Redress) of the Cartagena Protocol», Zurich, 22 March 2002. Document $n^{\circ} 20$ of Workshop hold in The Hague on 21 April 2002, on the occasion of COP - 6 by the Meridian Institute, Washington. Voir également les travaux de la Conference on Risk Perception, Liability and Insurability, qui a eu lieu du 3 au 4 November 2003, dans le cadre du International Biotech Forum, au Centre for Global Dialogue de Swiss Re, à Rüschlikon.

46 Commissariat Général du Plan, OGM et agriculture: option pour l'action publique, Paris, La documentation française, 2001, pp. 270-271.

47 Christian Babusiaux, Jean-Yves Le Déaut et al., Plantes transgéniques: 1'expérimentation est-elle acceptable?, Paris, La documentation française, 2003, p. 38. 
OGM. Autrement dit, les assurances insistent sur les notions de «mesurabilité » et «prévisibilité» des risques pour qu'elles puissent les appréhender et les assurer. En revanche, les conséquences fortuites, accidentelles de l'utilisation des OGM sont assurables, sous réserve de l'existence d'un cadre juridique en vigueur. Et précisément, le bât blesse sur ce dernier point, étant donné que dans la plupart des cas il manque un cadre législatif adapté aux problèmes qui peuvent surgir dans le domaine des OGM. Or, en l'absence d'une base légale qui prévoit l'attribution des responsabilités (RC) en cas d'accidents provenant de l'application des produits transgéniques, ou sans précision de la période de prescription, les assureurs ne peuvent pas agir. Actuellement, face au problème inextricable que posent les OGM, les Etats n'arrivent pas à trouver une parade satisfaisante pour harmoniser les points de vue de tous les acteurs sur les plans national et international. Toutefois, la Suisse, en adoptant la Loi fédérale sur l'application du génie génétique au domaine non humain (LGG) du 21 mars 2003 a donné l'exemple que l'on peut légiférer dans ce domaine. La loi mentionne le principe de précaution (Art. 2), les conditions d'autorisation de la dissémination expérimentale (Art. 6), la nécessité de la séparation des flux des produits (Art. 16), les procédures de recours (Art. 27), la responsabilité civile (Art.30), les dommages causés à l'environnement (Art. 31) et le délais de la prescription (Art. 32).

En conclusion quelques remarques s'imposent:

- Le principe de précaution émerge à ce stade de la réflexion, compte tenu de la situation de couverture restreinte des dommages liés aux risques des $\mathrm{OGM}^{48}$. En fait, la notion de principe de précaution survient dans une situation paradoxale. L'insécurité toujours plus élevée ressentie par la population au sujet des risques possibles (dont la probabilité peut être cependant minime) contraste avec l'affaiblissement de l'institution de l'assurance ${ }^{49}$. Dans ces conditions, le principe de précaution a plusieurs rôles à jouer dans le domaine des assurances. D'abord, les débats en cours attirent l'attention du public sur le fait que dans la société technico-scientifique la multiplication des catastrophes technologiques est toujours possible et que ce risque contribue à la fragilité des organisations socio-économiques complexes ${ }^{50}$. Cette complexité est renforcée par le fait qu'avec les biotechnologies nous avons à faire avec les organismes vivants qui constituent un système ouvert, qui sont en interaction constante avec leur milieu, qui survivent grâce à l'échange de matière et d'énergie avec leur environnement. Or, ce processus est indéterminé et son effet est aléatoire. En effet, des scientifiques s'interrogent également sur l'évolution future des plantes OGM, compte tenu du fait que l'évolution des

48 La Commission des communautés européennes considère que «l'invocation ou non du principe de précaution est une décision prise lorsque les informations scientifiques sont incomplètes, peu concluantes ou incertaines et lorsque les indices donnent à penser que les effets possibles sur la santé humaine, animale ou végétale pourraient être dangereux et incompatibles avec le niveau de protection choisi » (ibid., p. 17).

49 Demarcq, op. cit., p. 45.

so OCDE, Emerging Systemic Risks: A New Frontier? Presentation by Pierre-Alain Schieb, March $28^{\text {th }}, 2003$ at the Conference: Risikogesellschaft Wohin? OCDE International Futures Programme. 
génomes est instable. Séralini souligne que la «complexité cachée $d u$ vivant (...) peut provoquer des interactions métaboliques inattendues ${ }^{51}$. Il n'est pas étonnant dès lors que dans l'appréhension des risques liés aux organismes génétiquement modifiés reste en grande partie hypothétique.

- Une autre raison de l'incertitude qui entoure le développement et l'application des OGM découle du fait que les propriétés d'un gène ne peuvent être considérées isolément, mais doivent être considérées de manière contextuelle. Elles dépendent de leur environnement génétique et s'expriment en interrelation avec d'autres gènes d'ADN. On peut donc constater que les technologies du vivant sont des processus complexes, mal connus, d'où une certaine méconnaissance des risques.

- La mise en œuvre du principe de précaution interpelle tous les acteurs, scientifiques, décideurs politiques et économiques, membres de la société civile, pour les interroger sur la nature et le degré des risques que la communauté est disposée à accepter et à assumer. Le principe de précaution instaure non seulement un état de vigilance continuel pour guetter l'arrivée de nouveaux risques collectifs qui pourraient menacer, le cas échéant, la sécurité des personnes, leur santé et de l'environnement; il impose aussi un dialogue permanent avec toutes les parties concernées pour adapter les limites de la protection assurée en fonction de l'évolution rapide et mouvante des connaissances scientifiques et techniques. Le principe de précaution offre ainsi « de nouveaux repères dont les acteurs sociaux se saisissent pour réorganiser le jeu de la décision collective $»^{52}$.

- L'approche basée sur le principe de précaution associée à l'expérience acquise par les assurances et l'évaluation des risques en amont, permettrait une meilleure préparation de la prise de décision dans l'incertitude ${ }^{53}$. L'application du principe de précaution amènera vraisemblablement les assureurs à déplacer l'accent qu'ils mettent encore sur les conséquences de la réalisation des risques, vers l'anticipation des risques majeurs (en mettant en place une sorte de «early warning système»). Les assurances deviendraient alors des partenaires proactives dans l'élaboration des hypothèses lors de la mise en œuvre du principe de précaution.

51 Gilles-Eric Séralini, op. cit., parle de «mutagenèse par insertion», p. 257 (souligné par nous).

52 Olivier Godard, «Le principe de précaution: s'éloigner du positivisme scientifique pour civiliser les risques », in Cahiers français, $\mathrm{n}^{\circ}$ 294, « Science et société », Paris, La documentation française, janvier-février 2000, p. 14

53 Il est vrai que ce n'est pas la première fois que l'industrie de l'assurance se trouve devant le dilemme de devoir assurer une activité dont les risques sont inconnus. Elle connaît notamment la notion de «risque de développement» qui répond précisément à la situation de devoir envisager des risques qui ne sont pas connus au moment de la mise des produits sur le marché. En d'autres termes, «on entend par risque de développement les dommages dont l'éventualité ne pouvait être décelée en l'état de connaissances scientifiques et techniques en vigueur au moment où 1 es faits à l'origine du dommage ont été commis (Swiss Re)». L'assurabilité de ces risques se pose dans la pratique et d'une manière générale, le contrat d'assurance exclut ce risque, même s'il intervient dans le cadre d'exploitation normale des entreprises. Voir aussi les travaux de François Ewald à ce sujet. 
- Dans la situation d'incertitude, la conception de la responsabilité à l'égard des dommages se transforme aussi. Il semble que l'on va s'orienter de plus en plus vers un régime de responsabilité collective sans faute, au lieu de chercher à imputer individuellement une cause ou une faute qui serait à l'origine d'une catastrophe $^{54}$. En d'autres termes, on passe d'une attribution individuelle de la responsabilité à une socialisation de la responsabilité des dommages, étant donné qu'en cas de la contamination d'une parcelle non OGM par des semences OGM, il est certainement difficile d'identifier à qui incombe la faute, au semencier, au négociant ou à l'agriculteur voisin. Le rapport adressé au Ministère français de l'écologie et du développement durable, propose d'instaurer un principe de responsabilité objective sans faute en matière de dissémination de OGM», tout en étant conscient que la détermination de la faute reviendra à l'expertise. Le rapport en question note enfin, que la responsabilité administrative qui incomberait à l'Etat qui délivre l'autorisation de dissémination d'une plante transgénique devrait être examinée sous l'angle du principe de précaution en l'état actuel du droit ${ }^{55}$. On peut conclure provisoirement que la question est loin d'être tranchée et que le débat reste ouvert en matière de responsabilité civile face aux risques des OGM. Il reste encore un long chemin à parcourir avant de trouver des solutions juridiques, économiques et sociales satisfaisantes.

- Il n'en reste pas moins que d'une manière ou d'une autre, tôt ou tard, la collectivité sera appelée à fixer de nouvelles règles relatives à la nature et au degré de risque acceptable au cas en cas. Car «la responsabilité est un art de la décision, comme préalable à l'action» ${ }^{56}$. C'est dans ce sens que la clarification de la responsabilité civile peut contribuer au raisonnement en matière de précaution. "C'est en réalité un nouveau processus de décision qu'il faut inventer», écrit Laurence Engel ${ }^{57}$ qui ne saurait se borner au seul principe de précaution. Ce sont plutôt les principes de «bon gouvernement» applicables en matière d'évaluation et de choix technologique qu'il est urgent d'élaborer ${ }^{58}$. Ces principes nous invitent à évoluer vers l'établissement de règles codifiées qui prescriront une procédure d'alerte propre à devancer à temps les effets des risques technologique majeurs. La bonne gouvernance et l'application du principe de précaution préconisent ainsi à anticiper (anticipation prospective), à négocier (avec l'idée de la responsabilité comme fondement de toute décision) et, enfin, à réfléchir sur d'autres bases assurantielles. L'irréversibilité des nouveaux risques technologiques nous y convoque de manière urgente.

54 Dans le domaine des assurances, la responsabilité se définit comme l'obligation de réparer le dommage que l'on a causé. Les aspects juridiques de la responsabilité civile et des réparations des dommages (liability and redressement) sont exposés d'une manière approfondie dans ce volume par Philippe Cullet.

55 Christian Babusiaux, Jean-Yves Le Déaut, op. cit. pp. 37 -38

56 Laurence Engel, op. cit., p. 109. Cette forme de responsabilité est illustrée par la phrase souvent citée de Mme Georgina Dufoix, ancienne Ministre de la santé en France,: « responsable, mais pas coupable» au sujet de l'affaire du sang contaminé.

s7 Laurence Engel, op. cit., p. 109.

58 Bruno Latour, «Du principe de précaution au principe de bon gouvernement», Etudes, no 393-4, octobre 2000, pp. 339-346. 


\section{BIBLIOGRAPHIE}

Aronson Daniel, (1999), «Les assureurs tirent la sonnette d'alarme», Alternatives économiques, no 174 , octobre , pp. 46-48.

Audinot Jean-Pierre, (2002), «Assurance», in Encyclopaedia Universalis, CD-Rom, version 8.

Babusiaux Christian, Le Déaut Jean-Yves et al., (2003), Plantes transgéniques : l' expérimentation estelle acceptable?, Paris, La documentation française.

Baechler Jean, (1995), Le capitalisme, t. II, L'économie capitaliste, coll. Folio-histoire, Paris, Gallimard.

Beck Ulrich, (2001), Société du risque. Sur la voie d' une autre modernité, Paris, Aubier.

Beck Ulrich, (1993), «De la société industrielle à la société à risques», Revue suisse de sociologie, $\mathrm{n}^{\circ} 19$, pp. 311-337.

Bernstein Peter L. (1998), Plus forts que les dieux. La remarquable histoire du risque, Paris, Flammarion.

Boyer Robert, Chavance Bernard, Godard Olivier, (1991), Les figures de l'irréversibilité en économie, Paris, EHESS.

Briys Eric, De Varenne François, (2000), «1’000 years of risk management », Risk, janvier, pp. 47-48.

Callens Stéphane, (1997), Les maîtres de l'erreur. Mesure et probabilité au XIX siècle, Paris, PUF.

CNA (Conseil national de l'alimentation), Rapport et avis sur le principe de précaution et la responsabilité dans le domaine alimentaire, groupe présidé par Mme Marie-Odile Gailing, version adoptée le septembre 2001.

Commissariat Général du Plan, OGM et agriculture : option pour l'action publique, Paris, La documentation française, 2001.

Demarcq François, (1988), «Le risque technologique majeur», Problèmes économiques et sociaux, no 591 , septembre.

Dubois-Maury Jocelyne, Chaline Claude, (2002), Les risques urbains, Paris, Armand Colin.

Dupuy Jean-Pierre, (2002), Pour un catastrophisme éclairé. Quand l'impossible est certain, Paris, Seuil.

Durrer Alex, «Quand le risque catastrophe devient placement financier», Références, Journal de Genève, s.d.

Engel Laurence, (1995), La responsabilité en crise, Paris, Hachette.

«L'économie sociale entre étatisation et capitalisme», Cahiers français, no 221, mai-juin 1985.

Ewald François, (1996), Histoire de l'Etat Providence, Paris, Grasset.

Ewald François, (1997), «Le retour du malin génie. Esquisse d'une philosophie de la précaution », in Godard Olivier (dir.), Le principe de précaution dans la conduite des affaires humaines, Paris, INRA, pp. 99-126.

Ewald François, Gollier Christian, De Sadeleer Nicolas, (2001), Le principe de précaution, Paris, PUF.

Giarini Orio, Stahel Walter, (1990), Les limites du certain, Lausanne, Presse polytechnique et universitaire romande.

Giraud Pierre-Noël, (2001), Le commerce de promesses, Paris, Seuil.

Godard Olivier, Henry Claude, Lagadec Patrick et al., (2002), Traité des nouveaux risques, coll. Folioactuel, Paris, Gallimard.

- «Le principe de précaution: s'éloigner du positivisme scientifique pour civiliser les risques», in Cahiers français, $n^{\circ} 294$, «Science et société », Paris, La documentation française, janvier-février 2000, pp. 14-19.

Hunyadi Mark, «Pourquoi avons-nous besoin du raisonnement de précaution?», Esprit, n 8-9, aoûtseptembre 2003, pp. 139-162.

International Biotech Forum, Conference on Risk Perception, Liability and Insurability, 3-4 November 2003, Rüschlikon, Swiss Re, Centre for Global Dialogue.

Kourilsky Philippe, Viney Geneviève, (2000), Le principe de précaution, Paris, Odile Jacob. 
Lascoumes Pierre, (1996), «La précaution comme anticipation des risques résiduels et hybridation de la responsabilité», L'Année sociologique, $\mathrm{n}^{\circ} 46$, pp. 359-382.

Latour Bruno, (2000), «Du principe de précaution au principe du bon gouvernement», Etudes, $\mathrm{n}^{\circ} 3934$, octobre, pp. 339-346.

Loubergé Henri, (2000), «Development in risk and insurance economics: The past 25 years,» Chapitre 1 in Handbook of Insurance Economics, G. Dionne (Ed.), Kluwer Academic Publishers, Boston, 3-33.

Loubergé Henri, «An economist's view on risk perceptions for severe accidents », Geneva Papers on Risk and Insurance - Issues and Practice, 26 (No 3, July 2001), 452-458.

Maréchal Jean-Paul, (1991), Le prix du risque, Paris, Presses du CNRS.

Munich Re Group, Genetic Engineering: A Challenge for the Insurance Industry, s.d.

- Genetic Engineering Law, Liability Law and Liability Insurance, s.d.

November Valérie, (2002), Les risques du territoire, Berne, Peter Lang.

«Risque et précaution», Projet, numéro spécial, nº 261, mars 2000.

Ricoeur Paul, (1994), «Le concept de responsabilité. Essai d'analyse sémantique», Esprit, octobre, pp. $28-48$

Séralini Gilles-Eric, (2003), Génétiquement incorrect, Paris, Flammarion.

Stepczynski Marian, Wyler Michael, (1991), Guide bancaire et financier, Lausanne, L'Hebdo, 1991. Swiss Re, (1998), Le génie génétique et l'assurance RC. Le poids de l'opinion publique, Zurich, 1998.

- (1989), Périls de la nature et sinistres catastrophiques, Zurich.

- (1996), L'environnement, la responsabilité civile et l'assurance, Zurich.

- (2002), Rapport annuel et Rapport de gestion. 